\title{
Retraction Note to: High-dose therapy and autologous stem cell transplantation for relapsed or high-risk diffuse large B-cell lymphoma: a nationwide survey
}

\author{
Sung-Won Kim ${ }^{1} \cdot$ Yoshitaka Asakura $^{1,2} \cdot$ Kinuko Tajima $^{1} \cdot$ Toshiki Iwai $^{3} \cdot$ Hirofumi Taji $^{4} \cdot$ Takaaki Chou $^{5}$. \\ Yasuo Morishima $^{6,7} \cdot$ Junji Suzumiya $^{8} \cdot$ Hisashi Sakamaki $^{9} \cdot$ Ritsuro Suzuki $^{8,10} \cdot$ Takahiro Fukuda $^{1}$
}

Published online: 8 October 2020

(c) Japanese Society of Hematology 2020

\section{Retraction to: International Journal of Hematology (2020) 111:256-266 https://doi.org/10.1007/s1218 5-019-02772-1}

The Editor-in-Chief has retracted the original article following an investigation by the National Cancer Center Hospital that concluded that the research took place before ethical approval was given. All authors agree to this retraction.
Publisher's Note Springer Nature remains neutral with regard to jurisdictional claims in published maps and institutional affiliations.
The original article can be found online at https://doi.org/10.1007/ s12185-019-02772-1.

Sung-Won Kim

skim@ncc.go.jp

1 Department of Hematopoietic Stem Cell Transplantation, National Cancer Center Hospital, 5-1-1 Tsukiji, Chuo-ku, Tokyo 104-0045, Japan

2 Department of Hematology and Oncology, Okinawa Chubu Hospital, Uruma, Japan

3 Department of Hematology, Japanese Red Cross Kyoto Daiichi Hospital, Kyoto, Japan

4 Department of Hematology and Cell Therapy, Aichi Cancer Center Hospital, Nagoya, Japan

5 Department of Hematology and Oncology, Niigata Cancer Center Hospital, Niigata, Japan
6 Department of Promotion for Blood and Marrow Transplantation, Aichi Medical University School of Medicine, Nagakute, Japan

7 Division of Epidemiology and Prevention, Aichi Cancer Center Research Institute, Nagoya, Japan

8 Department of Oncology and Hematology, Shimane University Hospital, Izumo, Japan

9 Hematology Division, Tokyo Metropolitan Cancer and Infectious Disease Center, Komagome Hospital, Tokyo, Japan

10 Department of HSCT Data Management and Biostatistics, Nagoya University School of Medicine, Nagoya, Japan 